\title{
A new surgical approach to apical segment lung diseases, including carcinomas and inflammatory diseases
}

\begin{abstract}
A new surgical approach to lung cancer and inflammatory pulmonary diseases has been developed. This approach focuses on diseases located in the apical segments of the lung and showing invasion or severe adhesion to the apical thoracic wall or to vital organs near the thoracic dome (including superior sulcus tumors). The conventional posterolateral approach leaves the surgeon "blind" because it forces the surgeon to perform the operation looking up through a tube from the bottom. This limited view makes accurate assessment of the surrounding vital organs involved in these diseases almost impossible and also increases the risk of injury to adjacent vital organs. The incision in this new approach allows extensive retraction of the scapula to provide easier access to the posterior chest wall. Because the incision curves upward anteriorly, rather than downward as usual, it gives excellent exposure of the apical anterior thoracic region. In the few cases in which we have used this approach, we have found that the surgical field is in plain view and that the operation is consequently safer and easier. So far we have not encountered any complications, and we can recommend this approach with confidence. $(J$ ThORaC Cardiovasc Surg 1994;107:32-6)
\end{abstract}

Toshiki Tatsumura, MD, PhD, ${ }^{a}$ Hirofumi Sato, MD, ${ }^{\mathrm{c}}$ Akihiro Mori, MD, ${ }^{\mathrm{d}}$

Mikiya Miyazaki, MD, ${ }^{\text {e }}$ Shinji Koyama, MD, ${ }^{\text {b }}$ Masaru Tsujimoto, MD, ${ }^{\text {b }}$ and

Toshio Furuno, MD, Toyama, Yokohama, Kanazawa, and Takaoka, Japan

$\mathrm{V}_{\mathrm{a}}$ treating lung diseases in the apical segment of the upper lung lobe complicated with invasion into adjacent organs, particularly for the so-called Pancoast tumor, ${ }^{1}$ or the superior pulmonary sulcus tumor. One such approach was reported by Shaw, Paulson, and Kee. ${ }^{2}$ Because their approach is not much different from the conventional posterolateral approach, however, it does not offer many advantages in accessing a larger surgical field. Masaoka,

From the Departments of Emergency and Surgery, ${ }^{\mathrm{b}}$ Toyama Medical and Pharmaceutical University, Toyama; Yokohama Sakae Hospital, ${ }^{\mathrm{c}}$ Yokohama; Minamigaoka Hospital, ${ }^{d}$ Kanazawa; and Shakaihoken Takaoka Hospital, ${ }^{,}$Takaoka, Japan.

Received for publication Jan. 22, 1993

Accepted for publication May 3, 1993.

Address for reprints: Toshiki Tatsumura, MD, PhD, FCCP, FICA, Department of Emergency, Toyama Medical and Pharmaceutical University, 2630 Sugitani, Toyama 930-01, Japan.

Copyright 21994 by Mosby-Year Book, Inc.

$0022-5223 / 94 \$ 1.00+.10 \quad \mathbf{1 2} / \mathbf{1} / \mathbf{4 8 8 0 6}$
Ito, and Yasumitsu ${ }^{3}$ described an anterior approach for surgical treatment of lung cancer that provides better access to the anatomy of this region; however, even this approach is limited. Although it provides good exposure for tumors that have invaded frontal thoracic structures, it does not overcome the difficulties in approaching tumors that have invaded the posterior thoracic wall.

A new surgical approach developed by one of us (T.T.) provides a large surgical field and allows direct observation of the anatomy of the apical thoracic dome and supraclavicular and infraclavicular regions. This approach was designed to improve access both to tumors located at the superior sulcus and to those involved in the apical segment of the upper lung lobe and that have invaded the upper thoracic wall. In addition, this approach can be used to treat inflammatory diseases such as lung abscesses, in which severe pleural adhesion in the upper thoracic cavity would be more safely and easily separated if the involved structures, including the thoracic wall and important vessels, could be observed directly. To date this approach has been used in 14 cases of apical 

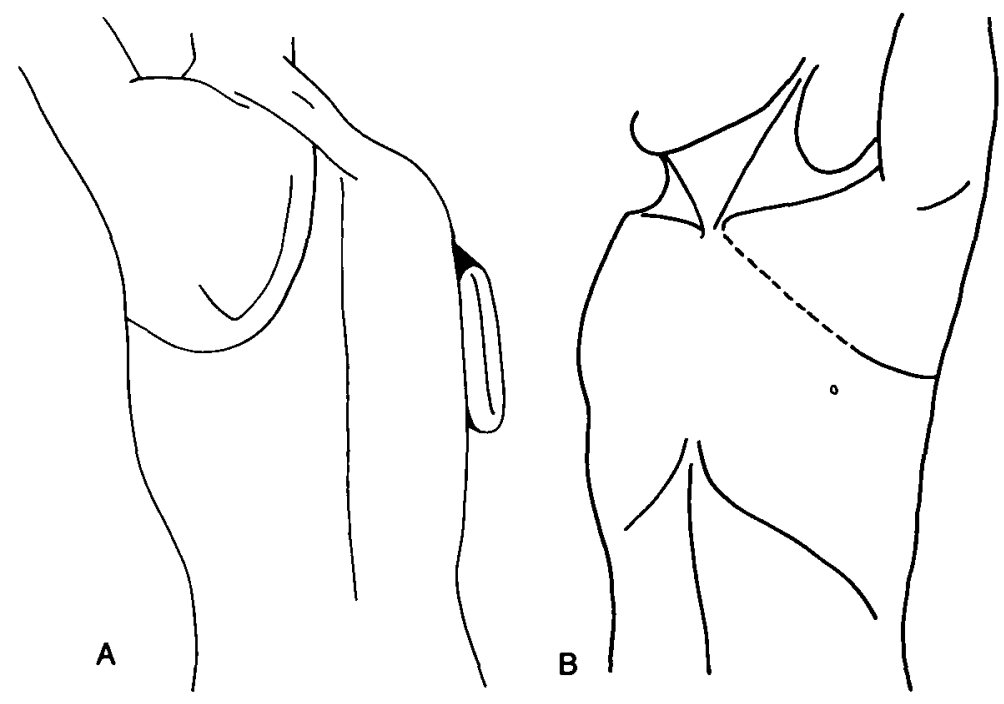

Fig. 1. Overview of the new approach. A, Semidorsal view of incisions. B, Semiventral view of incisions.

lung disease, with satisfactory results. There have been no complications, including complications associated with the upper arm of the involved side.

\section{Patients and methods}

Operative technique. The patient is placed and fixed with a Magic bag (Okada Medical Supply Corp., Tokyo, Japan) in a lateral position, with the diseased side facing up. The upper arm is straightened and supported by an arm board. A towel roll or other item is placed beneath the axilla, as in the preparation for a conventional thoracic operation. After the skin is well sterilized and dressed, an incision is begun at the level of the spinous process of the second or third thoracic vertebra and is continued downward along the paravertebral line and the scapula. The incision follows the tip of the angle of the scapula and continues upward to above the nipple level, ending at about the anterior axillary line (Fig. 1). The underlying muscles, such as the trapezius muscle, the rhomboid muscles, the latissimus dorsi muscle, and the serratus anterior muscle, are severed as they are encountered. This approach allows the scapula to be retracted high and away from the chest wall, providing a wide view of the posterior upper chest wall. Because the skin incision curves up anteriorly, at least to the anterior axillary line, the anterior portion of the incision can easily be retracted cranially and medially. In cases of apical lung diseases that are limited to the apical dome or anterior chest wall and do not invade the subclavian vessels, brachial plexus, and other structures of the supraclavicular region, incision to this level usually provides a wide and direct view of the surgical field of the anterior and posterior upper thoracic region. If desired, a wider view can be obtained by severing a portion of the proximal part of the greater and smaller pectoral muscles, to allow retraction to be extended cranially. To prevent injury to the trunk of the lateral and medial pectoral nerves during severing of the muscles, these muscles should be severed at the level of the rib because the nerves descend peripherally from the brachial plexus. Atrophic chang- es in the muscles during the late postoperative days can be avoided if these nerves are not severed. The thoracic cavity is usually opened through the third intercostal space, and the third rib is transected posteriorly close to the transverse process. It is sometimes necessary to transect the second and fourth ribs posteriorly to allow greater exposure of the thoracic cavity by means of a chest retractor.

In some cases the incision must continue beyond the anterior axillary line to allow evaluation of the extent of the diseases in the organs of the supraclavicular or infraclavicular regions. This is necessary in cases in which a true Pancoast tumor invades the adjacent surrounding tissues and organs at the level of the superior sulcus, such as a subclavian artery or vein, the brachial plexus, or other structures that may necessitate reconstruction of the vessels. This extended incision is especially needed in cases of more extensive disease. In such cases, a wider surgical field and a clearer view can be obtained by continuing the incision to the level of the sternoclavicular joint, with resection of the greater and smaller pectoral muscles near the ribs. Reapproximation of the resected pectoral muscles should be made before closure of the skin. This incision provides excellent access to the supraclavicular and infraclavicular regions. Further transection can be made at the cartilage level from the first to the third rib if the invasion of the lung cancer involves the organs of the superior sulcus region without involving the anterior bony thoracic wall. Special care should be taken to avoid injury to the internal thoracic artery during the anterior resection of the ribs. Furthermore, the ribs should be resected through the periosteal bed and the intercostal vessels and nerves should only be cut in the ribs with revealed tumor invasion and requiring bilateral resection, to prevent unnecessary complications in the ribs themselves. If both sides of the same rib are transected anteriorly and posteriorly during the procedure, either end (but preferably the anterior portion) should be joined to the anterior chest wall to prevent the paradoxic movement of the thoracic cage. In cases in which the tumor has invaded both the apical anterior chest wall and the organs of the superior sulcus, however, the 

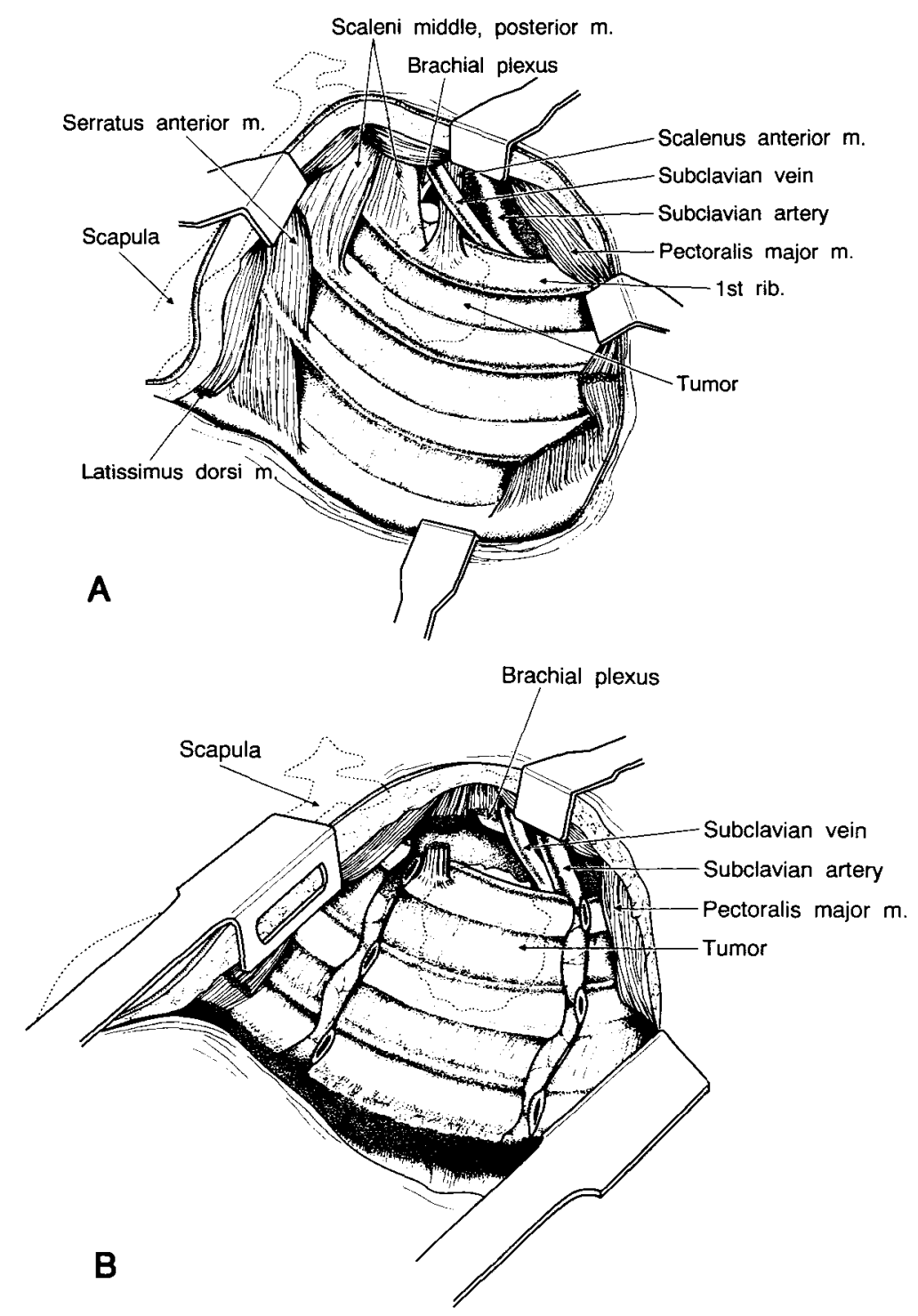

Fig. 2. Technique for approaching tumors located in apical lung. A, Pancoast tumor with invasion to apical pleura and apical anterior thoracic chest wall. Skin incision terminated at about midway between anterior axillary line and sternoclavicular joint, with partial resection of greater and smaller pectoral muscles. Superior sulcus and apical anterior thoracic structures are adequately exposed under direct view, and accurate assessment of the extent of invasion is possible. M., Muscle. Operation is easily performed at this level of incision if tumor invasion is limited to this area. B, First to third ribs, with a safety margin, have been resected bilaterally and freed from thoracic wall. Broken line signifies that the tumor incision is limited in the intrathoracic level of the chest wall; that is, the tumor has not infiltrated across the ventral part of the chest wall.

anterior portion of the first and other ribs should not be transected to secure wide exposure. Because the ribs have been infiltrated, both sides of each rib should be included in the resection, with care taken to maintain a safety margin of at least $2 \mathrm{~cm}$ from the tumor.

In cases of inflammatory diseases without chest wall resection, the thoracic cavity is closed layer by layer with interrupted sutures, so that the closure is airtight. In cases of lung cancer with resection of the chest wall, the reconstruction of the defect of the chest wall was done with Marlex mesh (Bard Implants, Billerica, Mass.). The mesh was folded into two layers and tightly secured to the cut edge of the bony chest wall and soft tissues.

Patients. The patients selected to undergo operation by this technique all had upper-segment lung diseases, such as lung cancer with invasion to the apical chest wall or involvement of 
the organs of the supraclavicular or infraclavicular region, or inflammatory diseases that revealed severe adhesions to the upper part of the thoracic wall and other vital organs, including the superior vena cava and the aorta.

There were seven patients with lung cancer in this series, five of whom showed invasion of the upper thoracic chest wall at levels ranging from the second to fourth ribs. Only two of these seven showed invasion into the apical dome structures of a so-called superior sulcus tumor. In one patient with invasion into the subclavian vessels, preoperative radiation was performed and the vessels were reconstructed.

The remaining seven patients had inflammatory parenchymal lung disease, all lung abscesses complicated with severe pleural adhesions to the apical thoracic chest wall and vessels. In all of these cases, the disease was resistant to medical management.

\section{Results}

The follow-up period for the cancer group ranged from 1 year 5 months to 6 years 1 month, with a mean of 4 years 2 months. In this group, five of the seven patients had invasion of the upper thoracic chest wall only. In these cases, the skin incision was terminated either at or slightly beyond the anterior axillary line, and resection of the greater pectoral muscle was minimized or avoided where possible. The affected arm showed no restriction in these cases. In the two patients (both male) with true superior sulcus tumor, the incision in one case extended to the sternoclavicular joint and in the other case the incision was terminated between the anterior axillary line and the joint. In both cases, the pectoral muscles were severed peripherally, at the costal to sternal attachment (patient 1 ) or at the costal margin (patient 2). These muscles were later reapproximated. Restricted movement of the shoulder was noted during the early postoperative days, especially in patient 1 , but this was primarily attributed to postoperative pain. To prevent shoulder stiffness, physical exercises were given to these two patients, beginning on the fifth postoperative day. These exercises included parts of the training program that we routinely apply to our patients who have undergone mastectomy. Exercises began with light flexion movements of the affected arm to about 45 to 60 degrees three times daily. Later, after all the drains had been taken out and the wound was completely healed, the training included flexion, abduction, and the tip of the hand reaching the opposite ear. The range of motion increased gradually, until it matched that of the unaffected arm. Full restoration of movement was achieved 3 months after operation for patient 1 and $1 \frac{1}{2}$ months after operation for patient 2 .

The follow-up period for the lung abscess group ranged from 7 months to 4 years 6 months, with a mean of 2 years 4 months. Because the skin incision in all cases was terminated at the anterior axillary line and only detachment of the inflamed adhesions and the lobectomies were performed, there were no restrictions of the arm or other complications. Moreover, no operative deaths were observed in association with this approach.

\section{Discussion}

Our limited experience with this approach indicates that only specific cases call for the skin incision to extend to the sternoclavicular joint to provide a wide surgical view of the supraclavicular and infraclavicular areas. Such an extensive incision is necessary in cases involving invasion into the subclavian artery and vein or into the brachial plexus, especially when the vessels require reconstruction. Our experience suggests that, if the tumor invasion is limited to the apical bony thoracic wall, excluding the subclavian vessels and other structures above the superior sulcus region, the skin incision can be terminated at the anterior axillary line or between the sternoclavicular joint; the pectoral muscles can be resected only partially (Fig. 2).

In this series, there have been no permanent, functional impairments in the movement of the arm on the affected side. The pectoral muscles do not atrophy after resection because the resection is carried out peripherally, at the costal or sternal margin of the muscles, preventing injury to the nerves. Similar observations have been made in the treatment of breast cancer. ${ }^{4}$ Finally, a daily regimen of physical exercise minimizes the risk of shoulder stiffness after operation through this approach.

The approach described by Paulson ${ }^{5}$ makes it difficult to assess the extent of a true Pancoast tumor, especially if the invasion has extended to adjacent organs, including the subclavian artery and vein, the brachial plexus, and the ribs. That technique also fails to produce a clearly visible surgical field, making the operation more difficult and increasing the risk of injury to nearby organs. The technique described here may offer a significantly better approach to diseases in the superior sulcus and upper thoracic regions.

For benign inflammatory diseases that show thick inflammatory adhesions to the apical region of the thorax, conventional thoracotomy does not allow the upper portion of the thoracic wall to be observed directly. Any manipulation must therefore be performed blind, which can sometimes cause unnecessary complications. During separation of the inflamed pleural adhesion, special care must be taken to avoid injuring the underlying organs, such as the subclavian vessels, the superior vena cava, the azygos vein, and the aorta. The approach described here allows the inflamed adhesion to be separated in plain view, thus avoiding the risk of injury to nearby organs.

Our experiences indicate that this approach is safer and 
easier to perform than traditional approaches. It therefore may be the method of choice for the surgical management of these pulmonary diseases.

\section{REFERENCES}

1. Pancoast HK. Superior pulmonary sulcus tumor: tumor characterized by pain, Horner's syndrome, destruction of bone and atrophy of hand muscles. JAMA 1932;99:1391-6.

2. Shaw RR, Paulson DL, Kee JL Jr. Treatment of the superior sulcus tumor by irradiation followed by resection. Ann Surg 1961;154:29-40.
3. Masaoka A, Ito Y, Yasumitsu T. Anterior approach for tumor of the superior sulcus. J THORAC CARDiovasC SuRG 1979;78:413-5.

4. Scanlon EF. The importance of the anterior thoracic nerves in modified radical mastectomy. Surg Gynecol Obstet 1981; 152:789-91.

5. Paulson DL. Technical considerations in stage III disease: the "superior sulcus" lesion. In: Delarue N, Eschapasse H, eds. International trends in general thoracic surgery, vol 1, lung cancer. Philadelphia: WB Saunders, 1985:121-31.

\section{Bound volumes available to subscribers}

Bound volumes of THE JouRnal of THORACIC AND CARDiovascular SURGERY are available to subscribers (only) for the 1994 issues from the Publisher, at a cost of $\$ 82.50$ for domestic, $\$ 106.28$ for Canadian, and $\$ 100.50$ for international subscribers for Vol. 107 (January-June) and Vol. 108 (July-December). Shipping charges are included. Each bound volume contains a subject and author index and all advertising is removed. Copies are shipped within 60 days after publication of the last issue of the volume. The binding is durable buckram with the Journal name, volume number, and year stamped in gold on the spine. Payment must accompany all orders. Contact Mosby-Year Book, Inc., Subscription Services, 11830 Westline Industrial Drive, St. Louis, Missouri 63146-3318, USA; phone 1 (800) 4534351 or (314) 453-4351.

Subscriptions must be in force to qualify. Bound volumes are not available in place of a regular JourNaL subscription. 